\title{
A CHARACTERISATION OF BERGMAN SPACES ON THE UNIT BALL OF $\mathbb{C}^{n}$
}

\author{
SONGXIAO LI \\ Department of Mathematics, Jiaying University, Guangdong, China \\ e-mail:lsx@mail.zjxu.edu.cn \\ HASI WULAN \\ Department of Mathematics, shantou university, Guangdong, China \\ e-mail:wulan@stu.edu.cn \\ RUHAN ZHAO \\ Department of Mathematics, SUNY, Brockport, NY 14420, USA \\ e-mail:rzhao@brockport.edu \\ and KEHE ZHU \\ Department of Mathematics, SUNY, Albany, NY 12222, USA \\ e-mail:kzhu@math.albany.edu \\ (Received 29 February 2008; accepted 17 November 2008)
}

\begin{abstract}
We obtain a new characterisation for weighted Bergman spaces $A_{\alpha}^{p}$ on the unit ball $\mathbb{B}_{n}$ of $\mathbb{C}^{n}$ in terms of a double integral of the functions $|f(z)-f(w)| /|z-w|$ and $|f(z)-f(w)| /|1-\langle z, w\rangle|$.
\end{abstract}

2000 Mathematics Subject Classification. 32A36.

1. Introduction. Let $\mathbb{B}_{n}$ denote the open unit ball in the $n$-dimensional complex Euclidean space $\mathbb{C}^{n}$. For $z=\left(z_{1}, \ldots, z_{n}\right)$ and $w=\left(w_{1}, \ldots, w_{n}\right)$ in $\mathbb{C}^{n}$ we write

$$
\langle z, w\rangle=z_{1} \bar{w}_{1}+\cdots+z_{n} \bar{w}_{n}, \quad|z|=\sqrt{\left|z_{1}\right|^{2}+\cdots+\left|z_{n}\right|^{2}} .
$$

Thus $\mathbb{B}_{n}=\left\{z \in \mathbb{C}^{n}:|z|<1\right\}$. When $n=1, \mathbb{B}_{n}$ is the open unit disc in the complex plane, and we will denote it by $\mathbb{D}$.

Let $d v$ denote the Lebesgue volume measure on $\mathbb{B}_{n}$. For any real parameter $\alpha$ we consider the weighted volume measure

$$
d v_{\alpha}(z)=\left(1-|z|^{2}\right)^{\alpha} d v(z)
$$

It is well known that $v_{\alpha}$ is a finite measure if and only if $\alpha>-1$ (see [16]). When $n=1$, we will use $d A_{\alpha}$ instead, because in this case we are dealing with weighted area measures. We emphasise here that we do not make the a priori assumption $\alpha>-1$ in this paper.

Suppose $0<p<\infty$ and $\alpha$ is real. We define the weighted Bergman space $A_{\alpha}^{p}$ as the space of $f \in H\left(\mathbb{B}_{n}\right)$ such that

$$
\left(1-|z|^{2}\right)^{N} \partial^{m} f(z) \in L^{p}\left(\mathbb{B}_{n}, d v_{\alpha}\right)
$$


for all multi-index $m=\left(m_{1}, \ldots, m_{n}\right)$ of non-negative integers satisfying $|m|=N$, where $N$ is any fixed non-negative integer with $p N+\alpha>-1$ and $H\left(\mathbb{B}_{n}\right)$ is the space of all holomorphic functions in $\mathbb{B}_{n}$. Here we use the standard notation that $|m|=m_{1}+\cdots+m_{n}$ and

$$
\partial^{m} f=\frac{\partial^{|m|} f}{\partial z_{1}^{m_{1}} \ldots \partial z_{n}^{m_{n}}} .
$$

It is well known that the space $A_{\alpha}^{p}$ thus defined is independent of the choice of $N$ (see [14] for a systematic study of $A_{\alpha}^{p}$ ).

We mention that if $\alpha>-1$, we can choose $N=0$, and the resulting spaces $A_{\alpha}^{p}$ are the usual weighted Bergman spaces with standard radial weight $\left(1-|z|^{2}\right)^{\alpha}$. If $\alpha=-(n+1)$, the spaces $A_{\alpha}^{p}$ coincide with the standard diagonal Besov spaces $B_{p}$ that have appeared in numerous places in the literature. Another special case that is worth mentioning is $A_{-1}^{2}$, which is nothing but the standard Hardy space $H^{2}$. It should be clear now what kind of spaces this paper is concerned with.

For any $f \in H\left(\mathbb{B}_{n}\right)$ we write

$$
L_{1} f(z, w)=\frac{|f(z)-f(w)|}{|1-\langle z, w\rangle|}\left(1-|z|^{2}\right)^{1 / 2}\left(1-|w|^{2}\right)^{1 / 2}
$$

and

$$
L_{2} f(z, w)=\frac{|f(z)-f(w)|}{|z-w|}\left(1-|z|^{2}\right)^{1 / 2}\left(1-|w|^{2}\right)^{1 / 2} .
$$

We can now formulate the main results of the paper as the following two theorems. The first one is valid for all dimensions but only in the case $\alpha>-1$. only if

Theorem A. Suppose $\alpha>-1, p>n+1+\alpha$, and $f \in H\left(\mathbb{B}_{n}\right)$. Then $f \in A_{\alpha}^{p}$ if and

$$
\int_{\mathbb{B}_{n}} \int_{\mathbb{B}_{n}}\left|L_{1} f(z, w)\right|^{p} d v_{t}(z) d v_{t}(w)<\infty,
$$

where $t=[\alpha-(n+1)] / 2$.

The second theorem is for the unit disc, but no restriction on $\alpha$ is required.

THEOREM B. Suppose $\alpha$ is real, $p>\max (-\alpha, \alpha+2)$ and $f$ is analytic in the unit disc. Then $f \in A_{\alpha}^{p}$ if and only if

$$
\int_{\mathbb{D}} \int_{\mathbb{D}}\left|L_{1} f(z, w)\right|^{p} d A_{t}(z) d A_{t}(w)<\infty
$$

if and only if

$$
\int_{\mathbb{D}} \int_{\mathbb{D}}\left|L_{2} f(z, w)\right|^{p} d A_{t}(z) d A_{t}(w)<\infty
$$

where $t=(\alpha-2) / 2$. 
Results of this type first appeared in [1], where it was shown that an analytic function $f$ in the unit disc $\mathbb{D}$ belongs to the Bloch space if and only if

$$
\sup \left\{\left|L_{2} f(z, w)\right|: z, w \in \mathbb{D}, z \neq w\right\}<\infty \text {. }
$$

This result was then generalised to higher dimensions in [5].

On the other hand, it was shown in $[\mathbf{1 0}]$ that an analytic function $f$ in the unit disc $\mathbb{D}$ belongs to the diagonal Besov space $B_{p}$ if and only if

$$
\int_{\mathbb{D}} \int_{\mathbb{D}}\left|L_{2} f(z, w)\right|^{p} d \tau(z) d \tau(w)<\infty
$$

where $p>2$ and $d \tau$ is the Möbius invariant area measure on $\mathbb{D}$. This result was then generalised to higher dimensions in [3].

2. The case of the unit ball. In this section we consider Bergman spaces in the unit ball of arbitrary dimension. When combined, the results of this section represent something more than Theorem A stated in the introduction. We begin with several preliminary estimates.

LEMMA 1. Let $r>0$, and let $D(z, r)$ denote the Bergman metric ball at $z$ with radius $r$. Then

$$
1-|z|^{2} \sim 1-|w|^{2} \sim|1-\langle z, w\rangle|
$$

for all $z \in \mathbb{B}_{n}$ and $w \in D(z, r)$. Furthermore, there exists a positive constant $C$ such that

$$
\left(1-|z|^{2}\right)^{p}|\nabla f(z)|^{p} \leq \frac{C}{\left(1-|z|^{2}\right)^{n+1}} \int_{D(z, r)}|f(z)-f(w)|^{p} d v(w)
$$

for all $z \in \mathbb{B}_{n}$ and all $f \in H\left(\mathbb{B}_{n}\right)$.

Proof. The first part is well known (see Lemma 2.27 of [16] for example).

To prove the second part, we use $|\widetilde{\nabla} f(z)|$ to denote the invariant holomorphic gradient of $f$ at $z$; that is

$$
|\widetilde{\nabla} f(z)|=\left|\nabla\left(f \circ \varphi_{z}\right)(0)\right|,
$$

where $\varphi_{z}$ is the involutive automorphism of the unit ball that interchanges the points 0 and $z$. It is well known that

$$
|\widetilde{\nabla}(f \circ \varphi)(z)|=|\widetilde{\nabla} f(\varphi(z))|
$$

for any holomorphic automorphism $\varphi$ of the unit ball (see [16] for more information about the Möbius invariant gradient).

It is easy to see (using Cauchy's estimate for example) that there exists a positive constant $C$ such that

$$
|\nabla f(0)|^{p} \leq C \int_{D(0, r)}|f(w)-f(0)|^{p} d v(w)
$$


for all $f \in H\left(\mathbb{B}_{n}\right)$. Replace $f$ by $f \circ \varphi_{z}$ and make a change of variables. Then

$$
|\widetilde{\nabla} f(z)|^{p} \leq C \int_{D(z, r)}|f(z)-f(w)|^{p} \frac{\left(1-|z|^{2}\right)^{n+1}}{|1-\langle z, w\rangle|^{2(n+1)}} d v(w)
$$

for all $f \in H\left(\mathbb{B}_{n}\right)$ and $z \in \mathbb{B}_{n}$. Combining this with the first part of the lemma, we can find another positive constant $C$ such that

$$
|\widetilde{\nabla} f(z)|^{p} \leq \frac{C}{\left(1-|z|^{2}\right)^{n+1}} \int_{D(z, r)}|f(z)-f(w)|^{p} d v(w)
$$

for all $f \in H\left(\mathbb{B}_{n}\right)$ and $z \in \mathbb{B}_{n}$. By Lemma 2.14 of [16], we always have

$$
\left(1-|z|^{2}\right)|\nabla f(z)| \leq|\widetilde{\nabla} f(z)|,
$$

so the desired result is proved.

LEMMA 2. The involutive automorphism $\varphi_{z}$ has the following properties:

$$
1-\left|\varphi_{z}(w)\right|^{2}=\frac{\left(1-|z|^{2}\right)\left(1-|w|^{2}\right)}{|1-\langle z, w\rangle|^{2}}
$$

and

$$
\left|\varphi_{z}(w)\right|^{2}=\frac{|z-w|^{2}+|\langle z, w\rangle|^{2}-|z|^{2}|w|^{2}}{|1-\langle z, w\rangle|^{2}} .
$$

Consequently,

$$
\left|\varphi_{z}(w)\right| \leq \frac{|z-w|}{|1-\langle z, w\rangle|}
$$

and

$$
\frac{\left(1-|z|^{2}\right)\left(1-|w|^{2}\right)}{|z-w|^{2}} \leq \frac{1-\left|\varphi_{z}(w)\right|^{2}}{\left|\varphi_{z}(w)\right|^{2}}
$$

and

$$
\frac{1}{|z-w|} \leq \frac{1}{\left|\varphi_{z}(w)\right||1-\langle z, w\rangle|}
$$

Furthermore, when $n=1$, all the inequalities above become equalities.

Proof. The first equality follows from (1.5) in Lemma 1.2 of [16], and the second equality follows from the first one and some elementary calculations. The first inequality follows from the second equality and the Cauchy-Schwarz inequality. The second inequality follows from the first inequality, while the third inequality follows from the first equality and the second inequality.

We now proceed to the proof of Theorem A.

Lemma 3. Suppose $p+\alpha>-1$ and $f$ is holomorphic in $\mathbb{B}_{n}$. If $\beta$ and $\gamma$ are real parameters such that

$$
\beta+\gamma=\alpha+p-(n+1)
$$


and if

$$
\int_{\mathbb{B}_{n}} \int_{\mathbb{B}_{n}} \frac{|f(z)-f(w)|^{p}}{|1-\langle z, w\rangle|^{p}} d v_{\beta}(z) d v_{\gamma}(w)<\infty
$$

then $f \in A_{\alpha}^{p}$.

Proof. Recall that $D(z, r)$ is the Bergman metric ball at $z$ with radius $r$, where $r$ is any fixed positive radius. By the second part of Lemma 1 , there exists a positive constant $C$ such that

$$
\left(1-|z|^{2}\right)^{p}|\nabla f(z)|^{p} \leq \frac{C}{\left(1-|z|^{2}\right)^{n+1}} \int_{D(z, r)}|f(w)-f(z)|^{p} d v(w) .
$$

Combining this with the first part of Lemma 1, we see that there is another constant $C>0$ such that

$$
\left(1-|z|^{2}\right)^{p}|\nabla f(z)|^{p} \leq \frac{C\left(1-\left|z^{2}\right|\right)^{p-\gamma}}{\left(1-|z|^{2}\right)^{n+1}} \int_{D(z, r)} \frac{|f(w)-f(z)|^{p}}{|1-\langle z, w\rangle|^{p}} d v_{\gamma}(w),
$$

and so

$$
\left(1-|z|^{2}\right)^{p}|\nabla f(z)|^{p} \leq \frac{C\left(1-\left|z^{2}\right|\right)^{p-\gamma}}{\left(1-|z|^{2}\right)^{n+1}} \int_{\mathbb{B}_{n}} \frac{|f(w)-f(z)|^{p}}{|1-\langle z, w\rangle|^{p}} d v_{\gamma}(w) .
$$

Since

$$
\alpha+p-\gamma-(n+1)=\beta
$$

we conclude that

$$
\int_{\mathbb{B}_{n}}\left(1-|z|^{2}\right)^{p}|\nabla f(z)|^{p} d v_{\alpha}(z) \leq C \int_{\mathbb{B}_{n}} \int_{\mathbb{B}_{n}} \frac{|f(z)-f(w)|^{p}}{|1-\langle z, w\rangle|^{p}} d v_{\beta}(z) d v_{\gamma}(w) .
$$

Taking $N=1$ in the proof of $A_{\alpha}^{p}$ shows that $f \in A_{\alpha}^{p}$.

Lemma 4. Suppose $\alpha>-1, f \in A_{\alpha}^{p}$ and $\beta$ and $\gamma$ satisfy (1). If, in addition,

$$
-1<\beta<p-(n+1), \quad-1<\gamma<p-(n+1),
$$

then

$$
\int_{\mathbb{B}_{n}} \int_{\mathbb{B}_{n}} \frac{|f(z)-f(w)|^{p}}{|1-\langle z, w\rangle|^{p}} d v_{\beta}(z) d v_{\gamma}(w)<\infty
$$

Proof. Fix $f \in A_{\alpha}^{p}$, and let $I$ denote the integral above. An elementary triangle type inequality then shows that we can find a positive constant $C$ such that

$$
I \leq C \int_{\mathbb{B}_{n}} \int_{\mathbb{B}_{n}} \frac{|f(z)|^{p}+|f(w)|^{p}}{|1-\langle z, w\rangle|^{p}} d v_{\beta}(z) d v_{\gamma}(w) .
$$

So the integral $I$ will be finite if each of the following two integrals is finite:

$$
I_{1}=\int_{\mathbb{B}_{n}} \int_{\mathbb{B}_{n}} \frac{|f(z)|^{p} d v_{\beta}(z) d v_{\gamma}(w)}{|1-\langle z, w\rangle|^{p}}
$$


and

$$
I_{2}=\int_{\mathbb{B}_{n}} \int_{\mathbb{B}_{n}} \frac{|f(w)|^{p} d v_{\beta}(z) d v_{\gamma}(w)}{|1-\langle z, w\rangle|^{p}} .
$$

By Fubini's theorem

$$
I_{1}=\int_{\mathbb{B}_{n}}|f(z)|^{p} d v_{\beta}(z) \int_{\mathbb{B}_{n}} \frac{d v_{\gamma}(w)}{|1-\langle z, w\rangle|^{p}} .
$$

By (1) and (2), we have

$$
\beta-\alpha=p-\gamma-(n+1)>0
$$

It follows from this and a standard integral estimate (see Proposition 1.4 .10 of [6] or Theorem 1.12 of [16]) that there exists another positive constant $C$ such that

$$
I_{1} \leq C \int_{\mathbb{B}_{n}} \frac{|f(z)|^{p} d v_{\beta}(z)}{\left(1-|z|^{2}\right)^{\beta-\alpha}}=C \int_{\mathbb{B}_{n}}|f(z)|^{p} d v_{\alpha}(z)<\infty .
$$

A similar argument shows that $I_{2}<\infty$. This completes the proof of the lemma.

We now combine Lemmas 3 and 4 to obtain one of our main results.

THEOREM 5. Suppose $\alpha>-1$ and $f$ is holomorphic in $\mathbb{B}_{n}$. If $\beta$ and $\gamma$ are real parameters satisfying (1) and (2), then $f \in A_{\alpha}^{p}$ if and only if

$$
\int_{\mathbb{B}_{n}} \int_{\mathbb{B}_{n}} \frac{|f(z)-f(w)|^{p}}{|1-\langle z, w\rangle|^{p}} d v_{\beta}(z) d v_{\gamma}(w)<\infty .
$$

Proof. Since $\alpha>-1$ and $p>0$, the assumption $p+\alpha>-1$ in Lemma 3 is fulfilled. The desired result then follows from Lemmas 3 and 4 .

COROllary 6. Suppose $\alpha>-1, p>n+1+\alpha$ and $f$ is holomorphic in $\mathbb{B}_{n}$. Then $f \in A_{\alpha}^{p}$ if and only if

$$
\int_{\mathbb{B}_{n}} \int_{\mathbb{B}_{n}}\left|L_{1} f(z, w)\right|^{p} d v_{t}(z) d v_{t}(w)<\infty,
$$

where $t=[\alpha-(n+1)] / 2$.

Proof. Let

$$
\beta=\gamma=\frac{p}{2}+t=\frac{\alpha+p-(n+1)}{2} .
$$

Then $\beta$ and $\gamma$ satisfy condition (1). Also, the condition $\beta>-1$ is equivalent to $p>$ $n-(\alpha+1)$, which clearly follows from the assumptions that $p>n+1+\alpha$ and $\alpha>-1$. Since $\gamma=\beta$, the condition $\gamma>-1$ is satisfied as well. Finally, the conditions $\beta<$ $p-(n+1)$ and $\gamma<p-(n+1)$ are each equivalent to $p>n+1+\alpha$, which is assumed to be true. So the desired result follows from Theorem 5 . 
3. The case of the unit disc. In this section we consider the special case $n=1$. We will be able to remove the assumption $\alpha>-1$ in several results of the previous section.

LEMMA 7. Suppose $n=1, \alpha<-1$ and $f$ is a function in $A_{\alpha}^{p}$ of the unit disc $\mathbb{D}$. Let $\beta$ and $\gamma$ be real parameters satisfying (1). If, in addition,

$$
-1<\beta<(p-3) / 2, \quad-1<\gamma<(p-3) / 2,
$$

then

$$
\int_{\mathbb{D}} \int_{\mathbb{D}} \frac{|f(z)-f(w)|^{p}}{|z-w|^{p}} d A_{\beta}(z) d A_{\gamma}(w)<\infty .
$$

Proof. It is easy to see that the assumptions on the parameters imply that $p>1$. By the integral representation for Bergman spaces (see Corollary 31 of [14]), there exists a function $g \in L^{p}\left(\mathbb{D}, d A_{\alpha}\right)$ such that

$$
f(z)=\int_{\mathbb{D}} \frac{g(u) d A_{-1}(u)}{1-z \bar{u}}, \quad z \in \mathbb{D},
$$

so

$$
\frac{|f(z)-f(w)|}{|z-w|} \leq \int_{\mathbb{D}} \frac{|g(u)| d A_{-1}(u)}{|1-z \bar{u}||1-w \bar{u}|} .
$$

Let $1 / p+1 / q=1$, and write

$$
1=a+b, \quad-1=-\frac{1-\epsilon}{q}-\left(\frac{1}{p}+\frac{\epsilon}{q}\right),
$$

where $a$ and $b$ are real and $\epsilon>0$, whose exact values are to be specified later. It follows from Hölder's inequality that the function $|f(z)-f(w)| /|z-w|$ is less than or equal to

$$
\left[\int_{\mathbb{D}} \frac{|g(u)|^{p}\left(1-|u|^{2}\right)^{-p\left(\frac{1}{p}+\frac{\epsilon}{q}\right)} d A(u)}{|1-z \bar{u}|^{p a}|1-w \bar{u}|^{p a}}\right]^{\frac{1}{p}}\left[\int_{\mathbb{D}} \frac{\left(1-|u|^{2}\right)^{-1+\epsilon} d A(u)}{|1-z \bar{u}|^{q b}|1-w \bar{u}|^{q b}}\right]^{\frac{1}{q}} .
$$

Denote the second integral above by $I$ and estimate it using the Cauchy-Schwarz inequality and Proposition 1.4.10 of [6] as follows:

$$
\begin{aligned}
I & \leq\left[\int_{\mathbb{D}} \frac{\left(1-|u|^{2}\right)^{-1+\epsilon} d A(u)}{|1-z \bar{u}|^{2 q b}}\right]^{1 / 2}\left[\int_{\mathbb{D}} \frac{\left(1-|u|^{2}\right)^{-1+\epsilon} d A(u)}{|1-w \bar{u}|^{2 q b}}\right]^{1 / 2} \\
& \leq C\left[\frac{1}{\left(1-|z|^{2}\right)^{2 q b-1-\epsilon}\left(1-|w|^{2}\right)^{2 q b-1-\epsilon}}\right]^{1 / 2} \\
& =\frac{C}{\left[\left(1-|z|^{2}\right)\left(1-|w|^{2}\right)\right]^{q b-(1+\epsilon) / 2}} .
\end{aligned}
$$

Here we are assuming that $b$ and $\epsilon$ satisfy

$$
2 q b-1-\epsilon>0
$$


which will be justified later. Thus $|f(z)-f(w)| /|z-w|$ does not exceed a constant multiple of

$$
\frac{1}{\left[\left(1-|z|^{2}\right)\left(1-|w|^{2}\right)\right]^{b-(1+\epsilon) /(2 q)}}\left[\int_{\mathbb{D}} \frac{|g(u)|^{p}\left(1-|u|^{2}\right)^{-p\left(\frac{1}{p}+\frac{\epsilon}{q}\right)} d A(u)}{|1-z \bar{u}|^{p a}|1-w \bar{u}|^{p a}}\right]^{\frac{1}{p}},
$$

and $|f(z)-f(w)|^{p} /|z-w|^{p}$ is less than or equal to a constant multiple of

$$
\frac{1}{\left[\left(1-|z|^{2}\right)\left(1-|w|^{2}\right)\right]^{p b-p(1+\epsilon) /(2 q)}} \int_{\mathbb{D}} \frac{|g(u)|^{p}\left(1-|u|^{2}\right)^{-p\left(\frac{1}{p}+\frac{\epsilon}{q}\right)} d A(u)}{|1-z \bar{u}|^{p a}|1-w \bar{u}|^{p a}} .
$$

So the integral

$$
J=\int_{\mathbb{D}} \frac{|f(z)-f(w)|^{p}}{|z-w|^{p}} d A_{\beta}(z) d A_{\gamma}(z)
$$

is dominated by

$$
\int_{\mathbb{D}} \int_{\mathbb{D}}\left(1-|z|^{2}\right)^{s}\left(1-|w|^{2}\right)^{t} d A(z) d A(w) \int_{\mathbb{D}} \frac{|g(u)|^{p}\left(1-|u|^{2}\right)^{-p\left(\frac{1}{p}+\frac{\epsilon}{q}\right)} d A(u)}{|1-z \bar{u}|^{p a}|1-w \bar{u}|^{p a}},
$$

where $s=\beta+p(1+\epsilon) /(2 q)-p b$ and $t=\gamma+p(1+\epsilon) /(2 q)-p b$. We use Fubini's theorem and Proposition 1.4.10 of [6] again to obtain the estimates

$$
\int_{\mathbb{D}} \frac{\left(1-|z|^{2}\right)^{s} d A(z)}{|1-z \bar{u}|^{p a}} \leq \frac{C}{\left(1-|u|^{2}\right)^{p a-2-\beta-p(1+\epsilon) /(2 q)+p b}}
$$

and

$$
\int_{\mathbb{D}} \frac{\left(1-|w|^{2}\right)^{t} d A(w)}{|1-w \bar{u}|^{p a}} \leq \frac{C}{\left(1-|u|^{2}\right)^{p a-2-\gamma-p(1+\epsilon) /(2 q)+p b}},
$$

where the requirements (for Proposition 1.4.10 of [6])

$$
\begin{gathered}
s=\beta+p(1+\epsilon) /(2 q)-p b>-1, \\
t=\gamma+p(1+\epsilon) /(2 q)-p b>-1, \\
p a-2-\beta-p(1+\epsilon) /(2 q)+p b>0
\end{gathered}
$$

and

$$
p a-2-\gamma-p(1+\epsilon) /(2 q)+p b>0
$$

are to be justified later. We deduce that

$$
J \leq C \int_{\mathbb{D}} \frac{|g(u)|^{p}\left(1-|u|^{2}\right)^{-p\left(\frac{1}{p}+\frac{\epsilon}{q}\right)} d A(u)}{\left(1-|u|^{2}\right)^{2 p(a+b)-4-\beta-\gamma-p(1+\epsilon) / q}} .
$$


Simplify the exponents of $\left(1-|u|^{2}\right)$ above using (1). The result is

$$
J \leq C \int_{\mathbb{D}}|g(u)|^{p} d A_{\alpha}(z)<\infty .
$$

It remains for us to show that it is possible to choose $a, b$ and $\epsilon>0$ so that conditions (4)-(9) are all satisfied. Because conditions (5)-(9) all involve strict inequalities, we may as well assume that $\epsilon=0$. In other words, if these conditions hold for $\epsilon=0$, then they will also hold when $\epsilon$ is a sufficiently small positive number. Therefore, we want to show that it is possible to choose $a$ and $b$ such that $a+b=1$ and

$$
\left\{\begin{array}{l}
2 q b-1>0 \\
\beta+\frac{p}{2 q}-p b>-1 \\
\gamma+\frac{p}{2 q}-p b>-1 \\
p-2-\beta-\frac{p}{2 q}>0 \\
p-2-\gamma-\frac{p}{2 q}>0
\end{array}\right.
$$

Using the relation $1 / p+1 / q=1$ we can change the above conditions to

$$
\left\{\begin{array}{l}
b>(p-1) /(2 p) \\
b<(2 \beta+p+1) /(2 p), \\
b<(2 \gamma+p+1) /(2 p), \\
\beta<(p-3) / 2 \\
\gamma<(p-3) / 2
\end{array}\right.
$$

The last two conditions are part of the assumptions of the theorem. It is easy to see that the conditions $\beta>-1$ and $\gamma>-1$ are equivalent to

$$
\frac{p-1}{2 p}<\frac{2 \beta+p+1}{2 p}, \quad \frac{p-1}{2 p}<\frac{2 \gamma+p+1}{2 p} .
$$

Therefore, if we choose any $b$ according to

$$
\frac{p-1}{2 p}<b<\min \left(\frac{2 \beta+p+1}{2 p}, \frac{2 \gamma+p+1}{2 p}\right),
$$

then all the requirements are satisfied. This completes the proof of the lemma.

LEMMA 8. Suppose $n=1, \alpha=-1$ and $p>1$. If $f \in A_{\alpha}^{p}$, then

$$
\int_{\mathbb{D}} \int_{\mathbb{D}} \frac{|f(z)-f(w)|^{p}}{|z-w|^{p}} d A_{\beta}(z) d A_{\gamma}(w)<\infty,
$$

where $\beta=\gamma=(p-3) / 2$.

Proof. Since $p>1$, we have $0<p+\alpha_{1}<p-1$ whenever $\alpha_{1}<-1$ and $\alpha_{1}$ is sufficiently close to -1 . Fix such an $\alpha_{1}$. Similarly, fix an $\alpha_{2}$ such that $0<p+\alpha_{2}<$ $2(p-1)$, which is equivalent to $-1<\alpha_{2}<p-2$. Then there is a unique $\theta \in(0,1)$ such that $-1=(1-\theta) \alpha_{1}+\theta \alpha_{2}$.

For $k=1,2$ let

$$
\beta_{k}=\gamma_{k}=\frac{\alpha_{k}+p-2}{2}
$$


Then $\beta_{k}+\gamma_{k}=\alpha_{k}+p-2$. We also have

$$
-1<\beta_{1}=\gamma_{1}<\frac{p-3}{2}
$$

and

$$
-1<\beta_{2}=\gamma_{2}<p-2
$$

and

$$
(1-\theta) \beta_{1}+\theta \beta_{2}=\frac{p-3}{2}=\beta .
$$

It follows from Lemmas 4 and 7 that the operator $T$ defined by

$$
T f=\frac{f(z)-f(w)}{z-w}
$$

maps $A_{\alpha_{k}}^{p}$ into $L^{p}\left(\mathbb{D} \times \mathbb{D}, d A_{\beta_{k}} \times d A_{\gamma_{k}}\right)$, where $k=1,2$. By complex interpolation, the operator $T$ maps the space

$$
\left[A_{\alpha_{1}}^{p}, A_{\alpha_{2}}^{p}\right]_{\theta}=A_{-1}^{p}
$$

(see Corollary 37 of [[14]]) into the space

$$
\left[L^{p}\left(\mathbb{D} \times \mathbb{D}, d A_{\beta_{1}} \times d A_{\gamma_{1}}\right), L^{p}\left(\mathbb{D} \times \mathbb{D}, d A_{\beta_{2}} \times d A_{\gamma_{2}}\right)\right]_{\theta},
$$

which, according to a classical theorem of Stein-Weiss (see [7]) concerning the complex interpolation of $L^{p}$ spaces with different weights, is the same as

$$
L^{p}\left(\mathbb{D} \times \mathbb{D}, d A_{\beta} \times d A_{\gamma}\right) .
$$

Therefore, $f \in A_{-1}^{p}$ implies that

$$
\int_{\mathbb{D}} \int_{\mathbb{D}} \frac{|f(z)-f(w)|^{p}}{|z-w|^{p}} d A_{\beta}(z) d A_{\gamma}(w)<\infty,
$$

where $\beta=\gamma=(p-3) / 2$. Combining this with Lemma 3, we obtain the desired result.

Note that a different interpolation argument can be given when $n=1, \alpha=-1$ and $p \geq$ 2 (which is stricter than required). In fact, in this case, we can also consider the operator $\Phi$ defined by

$$
\Phi(f)=\frac{f(z)-f(w)}{z-w}\left(1-|z|^{2}\right)^{1 / 2}\left(1-|w|^{2}\right)^{1 / 2} .
$$

We already know that $\Phi$ maps the Bloch space $\mathcal{B}$ of the unit disc into $L^{\infty}(\mathbb{D} \times \mathbb{D})$ (see [1]). Next we consider the case $p=2$, so that $\beta+\gamma=-1$. If

$$
f(z)=\sum_{n=0}^{\infty} a_{n} z^{n}
$$


is a function in $H^{2}=A_{-1}^{2}$ of the unit disc, then the integral

$$
I=\int_{\mathbb{D}} \int_{\mathbb{D}} \frac{|f(z)-f(w)|^{2}}{|z-w|^{2}} d A_{\beta}(z) d A_{\gamma}(w)
$$

can be evaluated as follows:

$$
\begin{aligned}
I & =\int_{\mathbb{D}} \int_{\mathbb{D}}\left|\frac{f(z)-f(w)}{z-w}\right|^{2} d A_{\beta}(z) d A_{\gamma}(w) \\
& =\int_{\mathbb{D}} \int_{\mathbb{D}}\left|\sum_{n=1}^{\infty} a_{n} \sum_{i+j=n-1} z^{i} w^{j}\right|^{2} d A_{\beta}(z) d A_{\gamma}(w) \\
& =\sum_{n=1}^{\infty}\left|a_{n}\right|^{2} \int_{\mathbb{D}} \int_{\mathbb{D}}\left|\sum_{i+j=n-1} z^{i} w^{j}\right|^{2} d A_{\beta}(z) d A_{\gamma}(w) \\
& =\sum_{n=1}^{\infty}\left|a_{n}\right|^{2} \sum_{i+j=n-1} \int_{\mathbb{D}}\left|z^{i}\right|^{2} d A_{\beta}(z) \int_{\mathbb{D}}\left|w^{j}\right|^{2} d A_{\gamma}(w) \\
& \sim \sum_{n=1}^{\infty}\left|a_{n}\right|^{2} \sum_{i+j=n-1} \frac{1}{(i+1)^{\beta+1}(j+1)^{\gamma+1}} .
\end{aligned}
$$

Consider the positive coefficients

$$
C_{n}=\sum_{i+j=n-1} \frac{1}{(i+1)^{\beta+1}(j+1)^{\gamma+1}}, \quad n \geq 1 .
$$

If we write

$$
C_{n}=\sum_{i=0}^{n-1} \frac{1}{\left(\frac{1}{n}+\frac{i}{n}\right)^{\beta+1}\left(1-\frac{i}{n}\right)^{\gamma+1}} \frac{1}{n}
$$

then it is clear that, for large $n$, the coefficient $C_{n}$ is comparable to

$$
\int_{0}^{1} \frac{d x}{x^{\beta+1}(1-x)^{\gamma+1}}
$$

which is a convergent integral, as the assumptions on $\beta$ and $\gamma$ easily imply that $-1<\beta<$ 0 and $-1<\gamma<0$. We conclude that

$$
\int_{\mathbb{D}} \int_{\mathbb{D}} \frac{|f(z)-f(w)|^{2}}{|z-w|^{2}} d A_{\beta}(z) d A_{\gamma}(w) \sim \sum_{n=1}^{\infty}\left|a_{n}\right|^{2},
$$

provided $\beta+\gamma=-1$. In particular, the operator $\Phi$ defined earlier maps $A_{-1}^{2}=H^{2}$ boundedly into $L^{2}\left(\mathbb{D} \times \mathbb{D}, d A_{-3 / 2} \times d A_{-3 / 2}\right)$. By complex interpolation, the operator $\Phi$ maps the space $\left[A_{-1}^{2}, \mathcal{B}\right]_{\theta}=A_{-1}^{p}$ (see Theorem 38 of [14]) boundedly into the space

$$
\left[L^{2}\left(\mathbb{D} \times \mathbb{D}, d A_{-3 / 2} \times d A_{-3 / 2}\right), L^{\infty}\right]_{\theta}=L^{p}\left(\mathbb{D} \times \mathbb{D}, d A_{-3 / 2} \times d A_{-3 / 2}\right),
$$


where $\theta \in(0,1)$ and

$$
\frac{1}{p}=\frac{1-\theta}{2}+\frac{\theta}{\infty}
$$

Equivalently, if $p>2$, then $f \in A_{-1}^{p}$ implies that

$$
\int_{\mathbb{D}} \int_{\mathbb{D}} \frac{|f(z)-f(w)|^{p}}{|z-w|^{p}} d A_{\beta}(z) d A_{\gamma}(w)<\infty,
$$

where $\beta=\gamma=(p-3) / 2$.

We now put the necessary pieces together to prove the other main result of the paper.

THEOREM 9. Suppose $n=1, \alpha$ is real, $p>\max (-\alpha, \alpha+2)$ and $f$ is analytic in the unit disc. Then $f \in A_{\alpha}^{p}$ if and only if

$$
\int_{\mathbb{D}} \int_{\mathbb{D}}\left[\frac{|f(z)-f(w)|}{|z-w|}\left(1-|z|^{2}\right)^{\frac{1}{2}}\left(1-|w|^{2}\right)^{\frac{1}{2}}\right]^{p} d A_{t}(z) d A_{t}(w)<\infty,
$$

where $t=(\alpha-2) / 2$.

Proof. Let

$$
\beta=\gamma=\frac{p}{2}+t=\frac{\alpha+p-2}{2}
$$

Then condition (1) is satisfied:

$$
\beta+\gamma=\alpha+p-2=\alpha+p-(n+1)
$$

Also, the assumption $p>-\alpha$ is equivalent to $\beta=\gamma>-1$. Finally, the assumption $p>$ $\alpha+2$ is equivalent to $\beta=\gamma<p-(n+1)$. The desired result is then a consequence of Lemmas 3, 7 and 8 and Corollary 6 .

As an example, we can take $\alpha=-2$ and $p>2$, so an analytic function $f$ in the unit disc belongs to $A_{-2}^{p}$ (which is the classical diagonal Besov space $B_{p}$ ) if and only if

$$
\int_{\mathbb{D}} \int_{\mathbb{D}}\left[\frac{|f(z)-f(w)|}{|z-w|}\left(1-|z|^{2}\right)^{\frac{1}{2}}\left(1-|w|^{2}\right)^{\frac{1}{2}}\right]^{p} d A_{-2}(z) d A_{-2}(w)<\infty,
$$

where

$$
d A_{-2}(z)=d \tau(z)=\frac{d A(z)}{\left(1-|z|^{2}\right)^{2}}
$$

is the Möbius invariant area measure on the unit disc. This is a theorem that was proved in [10].

4. Further remarks. We first make a general comparison between double integrals of the functions $L_{1} f(z, w)$ and $L_{2} f(z, w)$. 
Proposition 10. Suppose $\beta$ and $\gamma$ are real, $p>0$ and $n \geq 1$. Then there exists $a$ positive constant $C$ such that $I(f) \leq C J(f)$ for all $f \in H\left(\mathbb{B}_{n}\right)$, where

$$
I(f)=\int_{\mathbb{B}_{n}} \int_{\mathbb{B}_{n}} \frac{|f(z)-f(w)|^{p}}{|z-w|^{p}} d v_{\beta}(z) d v_{\gamma}(w)
$$

and

$$
J(f)=\int_{\mathbb{B}_{n}} \int_{\mathbb{B}_{n}} \frac{|f(z)-f(w)|^{p}}{|1-\langle z, w\rangle|^{p}} d v_{\beta}(z) d v_{\gamma}(w) .
$$

Proof. By Lemma 2,

$$
\frac{1}{|z-w|} \leq \frac{1}{\left|\varphi_{z}(w)\right||1-\langle z, w\rangle|}
$$

for all $z \neq w$, so

$$
\begin{aligned}
I(f) & =\int_{\mathbb{B}_{n}} d v_{\beta}(z) \int_{\mathbb{B}_{n}} \frac{|f(w)-f(z)|^{p}}{|w-z|^{p}} d v_{\gamma}(w) \\
& \leq \int_{\mathbb{B}_{n}} d v_{\beta}(z) \int_{\mathbb{B}_{n}} \frac{|f(w)-f(z)|^{p}}{\left|\varphi_{z}(w)\right|^{p}} \frac{d v_{\gamma}(w)}{|1-\langle z, w\rangle|^{p}} .
\end{aligned}
$$

Making the change of variables $w \mapsto \varphi_{z}(w)$ in the inner integral above according to Proposition 1.13 of [16], we obtain

$$
I(f) \leq \int_{\mathbb{B}_{n}} d v_{\alpha}(z) \int_{\mathbb{B}_{n}} \frac{\left|f \circ \varphi_{z}(w)-f \circ \varphi_{z}(0)\right|^{p}}{|w|^{p}} \frac{d v_{\gamma}(w)}{|1-\langle z, w\rangle|^{2(n+1+\gamma)-p}} .
$$

It is elementary to show that there exists a positive constant $C$ (independent of $f$ ) such that

$$
I(f) \leq C \int_{\mathbb{B}_{n}} d v_{\alpha}(z) \int_{\mathbb{B}_{n}} \frac{\left|f \circ \varphi_{z}(w)-f \circ \varphi_{z}(0)\right|^{p}}{|1-\langle z, w\rangle|^{2(n+1+\gamma)-p}} d v_{\gamma}(w) .
$$

Making the change of variables $w \mapsto \varphi_{z}(w)$ in the inner integral again, we obtain

$$
I(f) \leq C \int_{\mathbb{B}_{n}} \int_{\mathbb{B}_{n}} \frac{|f(z)-f(w)|^{p}}{|1-\langle z, w\rangle|^{p}} d v_{\beta}(z) d v_{\gamma}(w) .
$$

When $n=1$, the inequality in the proposition above can be reversed. In fact, in this case, we always have

$$
\frac{1}{|1-z \bar{w}|}=\frac{1}{|z-w|} \frac{|z-w|}{|1-z \bar{w}|} \leq \frac{1}{|z-w|} .
$$

This last inequality, however, is invalid in higher dimensions. To see this, take any $z \in \mathbb{B}_{n}-\{0\}$ and take $w=\varphi_{z}(u)$ for some $u \in \mathbb{B}_{n}$ with $|u|=|z|$ and $\langle z, u\rangle=0$. Then it is easy to verify that

$$
\frac{|z-w|}{|1-\langle z, w\rangle|}=\frac{|z|}{\sqrt{1-|z|^{2}}},
$$

which is clearly unbounded as $z$ approaches the unit sphere. 
Our second remark still concerns higher dimensions, for which so far we need the extra assumption that $\alpha>-1$. Although we do not know how to deal with general $\alpha$, we can improve our Theorem A to the case in which $\alpha \geq-(n+1)$. In fact, according to [3], Theorem $\mathrm{A}$ is valid for $\alpha=-(n+1)$ and $p$ large enough. Combining this with the $\alpha>-1$ case, with the help of an interpolation argument as used in the proof of Lemma 8, we conclude that Theorem A remains true whenever $\alpha \geq-(n+1)$, as long as $p$ is sufficiently large.

Our next remark concerns a generalisation of our results in a different direction. More specifically, for any $\theta \in(0,1)$ we consider the operator

$$
L_{\theta} f(z, w)=\frac{|f(z)-f(w)|}{|z-w|}\left(1-|z|^{2}\right)^{\theta}\left(1-|w|^{2}\right)^{1-\theta}
$$

or, somewhat differently,

$$
L_{\theta} f(z, w)=\frac{|f(z)-f(w)|}{|1-\langle z, w\rangle|}\left(1-|z|^{2}\right)^{\theta}\left(1-|w|^{2}\right)^{1-\theta} .
$$

Then for certain $\alpha$ we conjecture that there exists a positive cutoff constant $p_{0}$ (dependent on $\theta$ and $\alpha$ ) such that for $p>p_{0}$ and $f \in H\left(\mathbb{B}_{n}\right)$ we have $f \in A_{\alpha}^{p}$ if and only if

$$
\int_{\mathbb{B}_{n}} \int_{\mathbb{B}_{n}}\left|L_{\theta} f(z, w)\right|^{p} d v_{t}(z) d v_{t}(w)<\infty,
$$

where $t=[\alpha-(n+1)] / 2$ is the same as before. At least one such result can be found in the literature, namely a holomorphic function $f$ in $\mathbb{B}_{n}$ belongs to the Bloch space if and only if

$$
\sup \left\{\left|L_{\theta} f(z, w)\right|: z, w \in \mathbb{B}_{n}, z \neq w\right\}<\infty,
$$

where $L_{\theta} f(z, w)$ is the first version above. See [15] for a simple proof in the onedimensional case, and see [13] for the higher-dimensional case.

There are several other results in the literature that are related to our main results. For example, Lemma 4 follows from a result in [11]. In fact, if $f \in A_{\alpha}^{p}$, then by the proof of Theorem 13 in [11], there exists a non-negative continuous function $g \in L^{p}\left(\mathbb{B}_{n}, d v_{\alpha}\right)$ such that

$$
\frac{|f(z)-f(w)|^{p}}{|z-w|^{p}} \leq \frac{g(z)^{p}}{|1-\langle z, w\rangle|^{p}}+\frac{g(w)^{p}}{|1-\langle z, w\rangle|^{p}}
$$

for all $z$ and $w$ in $\mathbb{B}_{n}$ with $z \neq w$. The desired estimate is then a consequence of Fubini's theorem and Proposition 1.4.10 of [6] (or Theorem 1.12 of [16]).

Another interesting connection of our results to prior results in the literature concerns the so-called diagonal operator $\Delta$, which maps a holomorphic function $f(z, w)$ in the bidisc $\mathbb{D} \times \mathbb{D}$ to the analytic function $f(z, z)$ in the unit disc $\mathbb{D}$. More specifically, if

$$
\int_{\mathbb{D}} \int_{\mathbb{D}} \frac{|f(z)-f(w)|^{p}}{|z-w|^{p}} d A_{\beta}(z) d A_{\beta}(w)<\infty
$$


where $\beta=(\alpha+p-2) / 2>-1$ (which is equivalent to $p+\alpha>0$ or $p>-\alpha$ ), then the function

$$
g(z, w)= \begin{cases}(f(z)-f(w)) /(z-w), & z \neq w \\ f^{\prime}(z), & z=w\end{cases}
$$

belongs to the Bergman space $A_{\beta}^{p}$ of the bidisc $\mathbb{D} \times \mathbb{D}$. If we apply the diagonal operator to $g$, then by a well-known result (see [8] for example), the function $g(z, z)=f^{\prime}(z)$ belongs to $A_{2(\beta+2)-2}^{p}$ of the unit disc. Since $2 \beta+2=\alpha+p$, it follows that $f \in A_{\alpha}^{p}$.

The main problem that is left unanswered in the paper is whether or not Theorem B remains valid in higher dimensions for any real $\alpha$ and sufficiently large $p$. Here we want to convince the reader that the proof of Lemma 7 is strictly one-dimensional. In fact, if we tried the same idea, even if we used the more general Theorem 30 of [14] (instead of its simpler corollary), we would realise that we still had to use the integral representation

$$
f(z)=\int_{\mathbb{B}_{n}} \frac{g(u) d v_{-n}(u)}{1-\langle z, u\rangle} .
$$

After going through the same details, we would have arrived at the following 'theorem': Suppose $\alpha<-(n-1) p-1$ and $\beta+\gamma=\alpha+p-(n+1)$. If

$$
-1<\beta<\frac{2 p-n p-n-2}{2}, \quad-1<\gamma<\frac{2 p-n p-n-2}{2},
$$

then $f \in A_{\alpha}^{p}$ implies that

$$
\int_{\mathbb{B}_{n}} \int_{\mathbb{B}_{n}} \frac{|f(z)-f(w)|^{p}}{|z-w|^{p}} d v_{\beta}(z) d v_{\gamma}(w)<\infty .
$$

What is wrong with this analogue of Lemma 7 in higher dimensions is that there exist no parameters $\beta$ and $\gamma$ that satisfy the above conditions, unless $n=1$. In fact, for such $\beta$ and $\gamma$, we must have

$$
-2<\beta+\gamma<2 p-n p-n-2
$$

which implies $2 p>n p+n$. Obviously, this is is possible only when $n=1$.

ACKNOWLEDGEMENTS. Wulan is supported by NSF-China. Zhu is partially supported by NSF-USA

\section{REFERENCES}

1. F. Holland and D. Walsh, Criteria for membership of Bloch space and its subspace, BMOA, Math. Ann. 273 (1986), 317-335.

2. E. Kwon, A characterization of Bloch space and Besov space, J. Math. Anal. Appl. 324 (2006), 1429-1437.

3. S. Li and H. Wulan, Besov spaces on the unit ball of $\mathbb{C}^{n}$, Indian J. Math. 48 (2006), 177-186.

4. M. Nowark, Bloch and Möbius invariant Besov spaces on the unit ball in $\mathbb{C}^{n}$, Complex Var. 44 (2001), 1-12. 719-726.

5. G. Ren and C. Tu, Bloch spaces in the unit ball of $\mathbb{C}^{n}$, Proc. Am. Math. Soc. 133 (2005),

6. W. Rudin, Function Theory in the Unit Ball of $\mathbb{C}^{n}$ (Springer-Verlag, New York, 1980). 
7. E. Stein and G. Weiss, Interpolation of operators with change of measures, Trans. Am. Math. Soc. 87 (1958), 159-172.

8. M. Stessin and K. Zhu, Composition operators on embedded disks, J. Operator Theory 56 (2006), 423-449.

9. K. Stroethoff, On Besov-type space characterizations for the Bloch space, Bull. Austral. Math. Soc. 39 (1989), 405-420.

10. K. Stroethoff, The Bloch space and Besov spaces of analytic functions, Bull. Austral. Math. Soc. 54 (1996), 211-219.

11. H. Wulan and K. Zhu, Lipschitz type characterizations for Bergman spaces, Can. Math. Bull. (in press).

12. R. Yoneda, Characterizations of Bloch space and Besov spaces by oscillations, Hokkaido Math. J. 29 (2000), 409-451.

13. R. Zhao, A characterization of Bloch type spaces on the unit ball of $\mathbb{C}^{n}, J$. Math. Anal. Appl. 330 (2007), 291-297.

14. R. Zhao and K. Zhu, Theory of Bergman spaces in the unit ball, Mém. Soc. Math. Fr. (in press).

15. K. Zhu, Operator theory in function spaces, second edition (American Mathematical Society, Providence, RI, 2007).

16. K. Zhu, Spaces of holomorphic functions in the unit ball (Springer-Verlag, New York, 2005). 